\title{
Amniotic Fluid and Placental Membranes: Unexpected Sources of Highly Multipotent Cells
}

\author{
Sean V. Murphy, $\mathrm{PhD}^{1}$ Anthony Atala, MD ${ }^{1}$ \\ ${ }^{1}$ Wake Forest Institute for Regenerative Medicine, Wake Forest \\ University School of Medicine, Winston-Salem, North Carolina \\ Semin Reprod Med 2013;31:62-68

\begin{abstract}
Address for correspondence and reprint requests Anthony Atala, MD, Wake Forest Institute for Regenerative Medicine, Wake Forest University Health Sciences, Medical Center Boulevard, Winston-Salem, NC 27157 (e-mail: aatala@wakehealth.edu).
\end{abstract}

\begin{abstract}
Gestational tissue such as the placenta, placental membranes, and amniotic fluid are usually discarded following birth. Recently, researchers have identified gestational tissue as an untapped source of stem cells that are highly multipotent and possess potent immunosuppressive properties. Placental mesenchymal stem cells (MSCs), human amnion epithelial cells (hAECs), and amniotic fluid-derived stem cells (AFSCs) have been shown to differentiate into various cell types including adipogenic, osteogenic, myogenic, endothelial, pulmonary, neurogenic, hepatogenic, cardiac, and pancreatic lineages. Their immunomodulatory properties suggest that gestational stem cells may have an important application in the treatment of various inflammatory

\section{Keywords}

- placenta

- amniotic

- fluid

- membrane

- stem cells diseases such as graft versus host and autoimmune diseases. In clinical and preclinical studies, gestational stem cells have shown efficacy in the treatment of Crohn disease, lung disease, diabetes, repair of bone defects, heart disease, kidney disease, neural degeneration, and blood disorders. Stem cells derived from the placenta, placental membranes, and amniotic fluid are a valuable resource for the field of regenerative medicine.
\end{abstract}

In recent years, gestational tissue such as the placenta, placental membranes, umbilical cord, and amniotic fluid have been recognized as an untapped resource for the field of regenerative medicine. These tissues have been shown to be a rich source of highly multipotent stem cells with potent immunosuppressive properties that make these cells an exciting new tool for the treatment of disease. ${ }^{1-5}$ Human amniotic fluid has been used in prenatal diagnosis for $>70$ years. However, there is now evidence that amniotic fluid may have more utility than only as a diagnostic tool and may be a source of a powerful therapy for a multitude of congenital and adult disorders. Recently, researchers have isolated and characterized highly multipotent cells from the amniotic fluid and placental membranes. ${ }^{1,2}$

Amniotic fluid-derived stem cells (AFSCs) can be isolated from a small amount of the fluid obtained during amniocentesis, a procedure that is already performed in many pregnancies to screen for congenital abnormalities. Mesenchymal and epithelial stem cells can be obtained from the placenta and placental membranes, which are usually discarded fol- lowing birth (-Fig. 1). The advantage of these sources of stem cells is that there are no ethical and legal considerations associated with their collection and use. Cell culture experiments with these types of cells have provided evidence that they may have the potential to differentiate into various cell types including adipogenic, osteogenic, myogenic, endothelial, pulmonary, neurogenic, hepatogenic, cardiac, and pancreatic lineages $^{1,6-8}$ (-Fig. 2). The highly multipotent and antiinflammatory properties of these gestational stem cells suggest potential clinical applications for the treatment of diseases including Crohn disease, lung disease, diabetes, bone defects, heart and kidney disease, neurologic disorders, and blood disorders. ${ }^{9-13}$

\section{Developmental Biology of the Amniotic Fluid and Placenta}

Gastrulation is a major milestone in early postimplantation development. ${ }^{14}$ At about embryonic day 6.5 (E6.5), gastrulation begins in the posterior region of the embryo. Pluripotent
Issue Theme Stem Cells Helping Reproductive Medicine; Guest Editor, Carlos Simón, MD, PhD
Copyright (c) 2013 by Thieme Medical Publishers, Inc., 333 Seventh Avenue, New York, NY 10001, USA. Tel: +1(212) 584-4662.
DOI http://dx.doi.org/ 10.1055/s-0032-1331799. ISSN 1526-8004. 


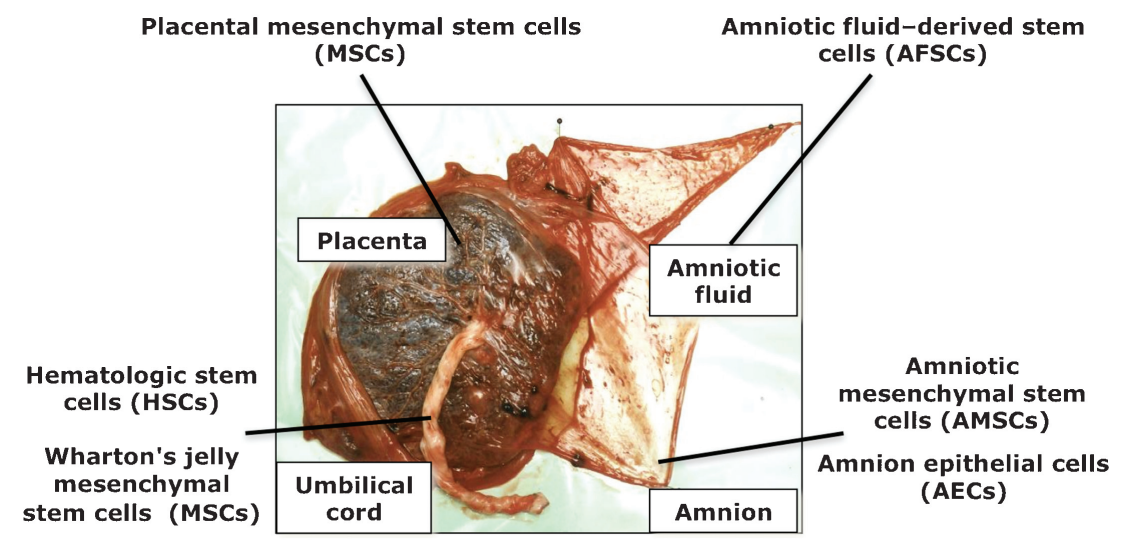

Figure 1 Gestational tissue as a source of stem cells. Cell types with therapeutic potential can be derived from gestational tissue including the placenta, umbilical cord, amniotic fluid, and the fetal membranes.
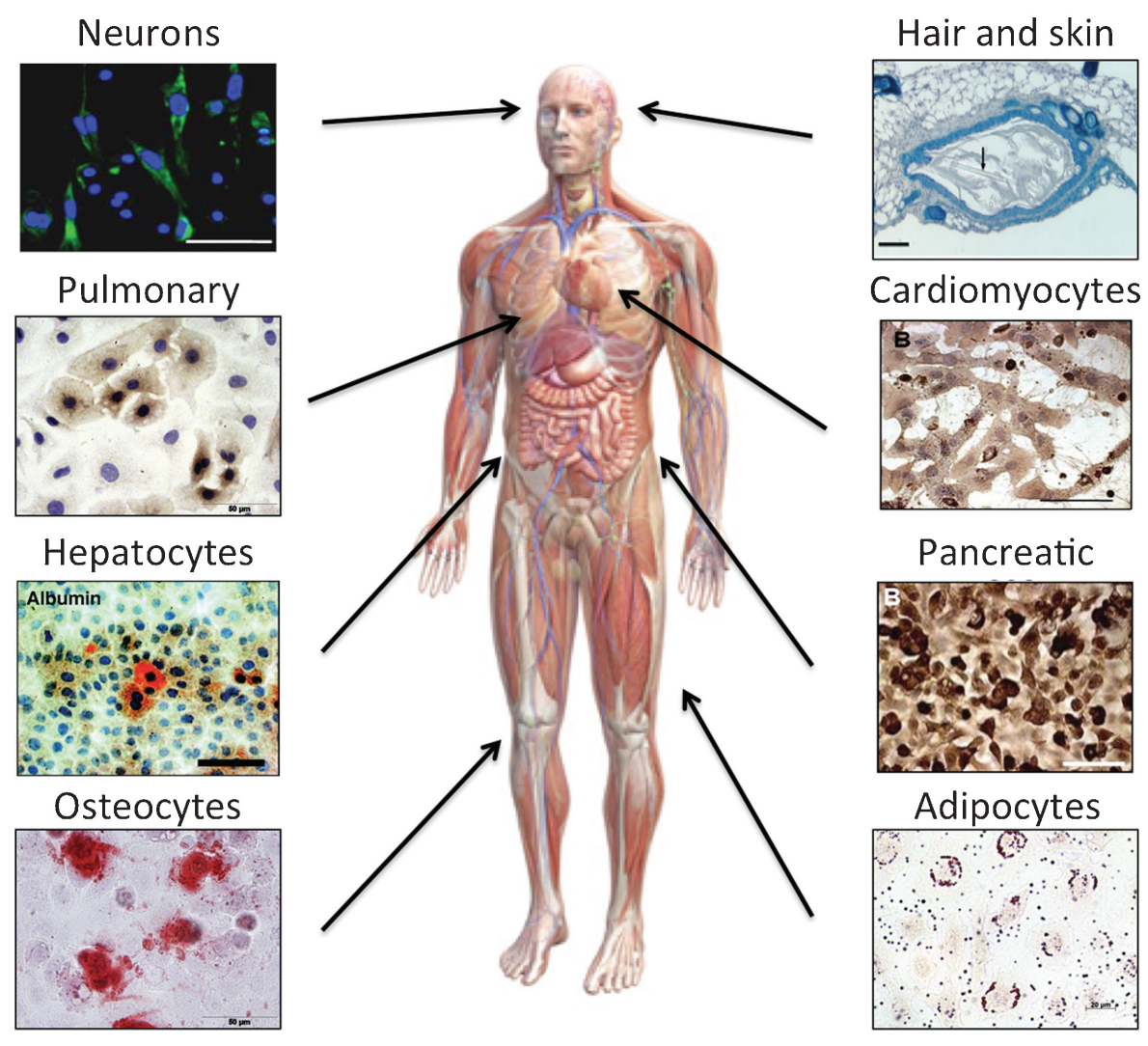

Figure 2 Multilineage differentiation of gestational stem cells. Stem cells derived from the amniotic fluid, placenta, and placental membranes have been differentiated into a diverse range of cell types, including but not limited to; neurons, hair and skin cells, pulmonary cells, cardiomyocytes, hepatocytes, pancreatic cells, osteocytes and adipocytes.

epiblast cells are allocated to the three primary germ layers of the embryo (ectoderm, mesoderm, and endoderm) and germ cells, which are the progenitors of all fetal tissue lineages, as well as to the extraembryonic mesoderm of the yolk sac, amnion, and allantois. ${ }^{15-18}$ The latter forms the umbilical cord as well as the mesenchymal part of the labyrinthine layer in the mature chorioallantoic placenta. ${ }^{15,19,20}$ The final positions of the fetal membranes result from the process of embryonic turning, which occurs around day 8.5 of gestation and pulls the amnion and yolk sac around the embryo. ${ }^{21,22} \mathrm{At}$ this time, the specification of tissue lineages is accomplished by the restriction of developmental potency and the activation of lineage-specific gene expression. 22,23 This process is strongly influenced by cellular interactions and signaling. ${ }^{24,25}$

The amniotic sac is a tough but thin transparent pair of membranes that holds the developing fetus until shortly 
before birth. The inner membrane, the amnion, contains the amniotic fluid and the fetus. The outer membrane, the chorion, envelops the amnion and is part of the placenta. $21,26,27$ The amnion is derived from ectoderm and mesoderm, and as it grows, it begins to fill with fluid. ${ }^{27}$ Originally this fluid is isotonic, containing proteins, carbohydrates, lipids, phospholipids, urea, and electrolytes. Later, urine excreted by the fetus increases its volume and changes its composition. ${ }^{28-31}$ The presence of the amniotic fluid ensures symmetrical structure development and growth. In addition, it cushions and protects the embryo, helps maintain consistent pressure and temperature, and permits freedom of fetal movement, which is important for musculoskeletal development and blood flow. ${ }^{32}$ The fetus can breathe in the amniotic fluid, allowing normal growth and development of the lungs. The fetus also swallows amniotic fluid, which contributes to gastrointestinal tract development, and as a result, components of the fluid pass via the fetal blood into the maternal blood. Amniotic fluid also contains a mixture of different cell types. Several different origins have been suggested for these cells. ${ }^{33}$ The presence of a Y chromosome in cell lines derived from cases in which the amniocentesis donor carried a male child implies that amniotic fluid cells originate from the developing fetus, sloughed from the fetal amnion membrane and skin, as well as the alimentary, respiratory, and urogenital tracts.

\section{Cells Derived from Amniotic Fluid and Placenta}

\section{Placental Mesenchymal Cells}

Mesenchymal stromal cells (MSCs) were initially identified in the bone marrow of adult subjects. ${ }^{34}$ MSCs contribute to the regeneration of mesenchymal tissues such as bone, cartilage, muscle, ligament, tendon, adipose, and stroma. MSCs have also been derived from other nonmarrow tissues such as adipose tissue, adult muscle, dental pulp, and gestational tissue. Within the last decade, several groups published relatively simple protocols for isolating a nonspecific population of cells with mesenchymal-like characteristics from the amniotic fluid and placenta. ${ }^{35-37}$ MSCs have the ability to differentiate into osteocytes, chondrocytes, and adipocytes in culture and possess immunomodulatory properties. These criteria are commonly used for defining bone-marrow MSCs:

- Adherence to plastic

- Formation of fibroblast colony-forming units

- Differentiation potential toward one or more mesodermal lineages

- Express CD90, CD73, and CD105

- Do not express CD45, CD34, CD14, or HLA-DR

Cells fitting these criteria have been isolated from the amniotic fluid, ${ }^{36}$ amnion, ${ }^{38}$ and chorion membranes ${ }^{39}$ as well as from the amniotic fluid. ${ }^{26}$ Steigman and Fauza described a method of isolating MSCs from the placenta where the fetal placental specimen is mechanically separated from the maternal decidua, either by blunt dissection or during the process of chorionic villus sampling. ${ }^{36}$ The remaining mesen- chymal core is minced and digested with a collagenase/ dispase mixture, filtered and plated on cover slips. Placental MSCs share with adult MSCs a spindle-shaped fibroblast appearance, the ability to adhere to plastic and to expand ex vivo, which are essential properties for isolation. However, placental MSCs expand quicker in vitro than adult MSCs and appear to be both less immunogenic and more immunosuppressive than their adult counterpart. ${ }^{40,41}$ These properties make placental MSCs attractive as a potential clinical therapy for inflammatory diseases.

\section{Amnion Epithelial Cells}

A multipotent epithelial cell population has also been isolated from the amnion membrane. ${ }^{2,42}$ Human amnion epithelial cells (hAECs) have attracted attention as a potential source of cells for regenerative therapies, ${ }^{35}$ with reports that epithelial cells derived from human term amnion possess multipotent differentiation ability, ${ }^{6}$ low immunogenicity, ${ }^{39}$ and anti-inflammatory functions. ${ }^{43}$ With a view to future clinical applications, our group demonstrated that hAECs can be collected, isolated, and stored in a manner suitable for clinical therapies. ${ }^{2}$ Term human amnion membrane is manually separated from the chorion membrane and washed in a saline solution to remove blood. Epithelial cells are isolated from the amnion by enzymatic digestion in Trypzean, an animal product-free recombinant trypsin. Approximately 120 million viable epithelial cells can be isolated from a single amnion membrane, and these cells can be maintained in serum-free culture conditions and display a normal karyotype and cell cycle distribution following culture while maintaining long telomere lengths. Although hAECs lack the proliferative capacity of placental MSCs, they are highly multipotent, differentiating into cells representing all three germ layers, specifically osteocytes, adipocytes, neurons, lung epithelial cells, cardiomyocytes, myocytes, hepatocytes, and pancreatic cells. ${ }^{2,6}$ The immunosuppressive properties of hAECs have been well characterized both in vitro ${ }^{44}$ and in vivo. ${ }^{43}$

It has been proposed that soluble factors produced by hAECs have anti-inflammatory effects and act to inhibit both the innate and adaptive immune systems. Thus hAECs may have important applications as a clinical cell therapy for a multitude of congenital and adult disorders.

\section{Amniotic Fluid Stem Cells}

AFSCs can be obtained from a small amount of fluid during amniocentesis at the second trimester. There is also the potential to collect amniotic fluid at term from routine cesarean deliveries. Kaviani and coworkers reported that just $2 \mathrm{~mL}$ of amniotic fluid contains up to 20,000 cells, $80 \%$ of which are viable. ${ }^{45}$ A highly multipotent subpopulation of AFSCs present in the amniotic fluid and placenta can be isolated through positive selection for cells expressing the membrane receptor c-kit (CD117). ${ }^{1}$ C-kit is expressed on a variety of stem cells including embryonic stem cells (ESCs), ${ }^{46}$ primordial germ cells, and many somatic stem cells. ${ }^{47}$ Approximately $1 \%$ of cells present in amniotic fluid have been shown to be c-kit positive. Progenitor cells maintain a round shape for 1-week after isolation when cultured in nontreated 
culture dishes. In this state, they demonstrate low proliferative capability. After the first week the cells begin to adhere to the plate and change their morphology, becoming elongated and proliferating rapidly. AFSCs show a high self-renewal capacity and maintain a normal karyotype at late passages and display normal G1 and G2 cell cycle checkpoints. They also conserve a long telomere length in late passages due to continued telomerase activity. Like ESCs, AFSCs form embryoid bodies in vitro that stain positive for markers of all three germ layers. However, unlike embryonic stem cells, when implanted into immunodeficient mice in vivo, AFSCs do not form teratomas, an essential safety characteristic for a potential cell therapy. AFSC have a high clonal capacity demonstrated using a technique involving retrovirally tagged cells. In this assay, a tagged single cell gave rise to a population that differentiated along six distinct lineages from all three germ layers: adipogenic, osteogenic, myogenic, endothelial, neurogenic, and hepatic.

\section{Amniotic Fluid and Placenta for Cell Therapy}

The highly multipotent and immunosuppressive cell populations that can be isolated from the amniotic fluid and placental tissue are a valuable source of cells that can be utilized for the treatment of disease. Here we discuss current clinical and preclinical applications of amniotic fluid and placental stem cells.

\section{Therapy for Crohn Disease}

Crohn disease, also known as regional enteritis, is a chronic inflammatory condition of the gastrointestinal tract. In patients with Crohn disease, the body's own immune system attacks the gastrointestinal tract resulting in abdominal pain, diarrhea, and vomiting as well as other nongastrointestinal symptoms. Traditional treatment for Crohn disease has focused on nonspecific anti-inflammatory or immunosuppressive agents. A considerable proportion of patients develop clinically significant side effects, require surgery or become unresponsive to therapy. A growing body of evidence indicates that placental MSCs possess immunomodulatory properties and may play specific roles as immunomodulators in the maintenance of peripheral tolerance, transplantation tolerance, and autoimmunity as well as in fetal-maternal tolerance. Celgene Cellular Therapeutics has developed a placental MSC therapy for treatment-resistant Crohn disease. ${ }^{48}$ In a phase 1 clinical trial, 12 patients with active moderate to severe treatment-resistant Crohn disease received two infusions of placental MSCs. All patients showed signs of clinical remission of the disease. Infusion of placental MSCs appeared to be safe, and no major toxicities were observed. Celgene Cellular Therapeutics plans to continue with phase 2 studies to treat Crohn disease as well to investigate the other potential applications including a therapy for ischemic stroke.

\section{Lung Regeneration}

Respiratory disease is a leading cause of morbidity and mortality. The causes of respiratory diseases differ, but the end resultant organ damage is similar-chronic inflammation, fibrosis, and scarring-leading to loss of functional lung tissue. Amnion epithelial cells show a potential to be utilized in the treatment of diseases such as cystic fibrosis, acute respiratory distress syndrome, chronic obstructive lung disease, pulmonary fibrosis, pulmonary edema, and pulmonary hypertension. In animal models of lung disease, the transplantation of hAECs has been shown to reduce both inflammation and subsequent fibrosis as well as improve lung function. ${ }^{10}$ Administration of hAECs to mice with bleomycin-induced lung disease resulted in decreased gene expression of proinflammatory cytokines tumor necrosis factor- $\alpha$, transforming growth factor- $\beta$, interferon- $\gamma$, and interleukin-6, reduced pulmonary collagen deposition, $\alpha$-smooth muscle actin expression, and inflammatory cell infiltrate. Our group recently demonstrated that hAECs interact with host macrophages to exert their reparative influence, possibly by inducing macrophages into an alternatively activated phenotype. ${ }^{49}$ Recent studies have also induced hAECs to express lung-specific proteins ${ }^{6}$ including the ion channel cystic fibrosis transmembrane conductance regulator, suggesting an important application of hAECs for the treatment of patients with cystic fibrosis. These important studies highlight the potential of hAECs for clinical and bioengineering applications for patients with lung disease.

\section{Pancreatic Tissue Insulin Production}

Current treatment for diabetes mellitus relies on daily multiple insulin injections or insulin pump placement, or $\beta$-cell or whole pancreas replacement. Islet transplantation is limited by the scarcity of transplant material and the requirement for lifelong immunosuppressive therapy. An alternative cellbased therapy would represent a major breakthrough in the management of this common chronic disorder. Wei and coworkers demonstrated that hAECs can be stimulated to expressed insulin and GLUT-2 mRNA, and they investigated the potential for hAEC to restore blood glucose levels in diabetic mice. ${ }^{50}$ In mice receiving hAECs, blood glucose decreased to normal levels posttransplantation. The body weights of hAEC-treated mice also normalized compared with mice not receiving cells. Chang et al demonstrated that placenta-derived MSCs can also be induced to secrete insulin and glucagon. ${ }^{51}$ They demonstrated that transplanted placenta-derived MSCs form glandlike tissues, differentiate into insulin and glucagon-positive cells, and stably restore normoglycemia in diabetic mice. These studies present a strong case for the use of gestational stem cells for the treatment of diabetes mellitus.

\section{Bone Regeneration}

Cell-based therapy for bone regeneration is an emerging technology. AFSCs have the potential to be utilized to treat craniofacial bone defects and spinal or major bone injury. Preclinical studies have established that three-dimensional scaffolds containing AFSC can generate highly mineralized bone tissue 8 weeks following transplantation into mice. ${ }^{1}$ Micro computed tomography scanning analysis of constructs 
at 18 weeks postimplantation confirmed the presence of hard tissue within the AFSC-seeded constructs. The density of the tissue-engineered bone found at the sites of implantation was found to be somewhat greater than that of mouse femoral bone. Scaffolds can be designed to produce bone to generate specific craniofacial shapes or at densities to facilitate the replacement of major bones damaged by car accidents or battle injuries. Although early in development, these studies demonstrate that AFSCs are a valuable tool for future therapies for bone regeneration.

\section{Myocardial Infarction}

Myocardial infarction causes tissue death, and the ability to replace that lost myocardial tissue is an important goal in the field of regenerative medicine. The therapeutic potential of AFSCs for acute myocardial infarction was demonstrated by Bollini et al in a study in which Wistar rats underwent 30 minutes of ischemia by ligation of the left anterior descending coronary artery, followed by administration of AFSCs. ${ }^{52}$ In this model AFSCs were shown to be cardioprotective, improving myocardial cell survival and decreasing the infarct size. Other studies have identified iron oxide particle-labeled AFSCs in the mouse heart by high-resolution magnetic resonance imaging up to 28 days following injection. ${ }^{12}$ Lee and coworkers used AFSCs to form spherically symmetrical cell bodies, which were xenogenically transplanted in the periinfarct area of an immune-suppressed rat via direct intramyocardial injection. ${ }^{53}$ The functional benefits of cell transplantation included the attenuation of the progression of heart failure, improved the global function, and increased the regional wall motion.

\section{Renal Disease}

Regenerative therapy has the potential to cure certain hereditary forms of kidney disease and acute kidney injury, and it could eliminate the need for dialysis and/or kidney transplants in some patients with end-stage kidney disease. Perin and coworkers demonstrated that AFSCs can contribute to renal development both ex vivo and in vivo. ${ }^{13,54}$ In a mouse model of acute tubular necrosis, AFSCs integrate into the damaged tubules and provide a protective effect, ameliorating tubular necrosis in the acute injury phase. AFSCtreated animals showed decreased creatinine and blood urea nitrogen blood levels and a decrease in the number of damaged tubules. This beneficial effect with AFSCs was also correlated with significant increases in proliferative activity of tubular epithelial cells, decreased cast formation, and decreased apoptosis of tubular epithelial cells. Integrated cells expressed PAX2, NPHS1 Dolicholus Biflorus, and Peanut Agglutinin indicating AFSCs are able to commit toward renal differentiation in vivo. These studies also show evidence of potent immunomodulatory effects of AFSCs that appear to influence the local immune response to prevent or promote the resolution of tissue damage.

\section{Neural Regeneration}

A major goal of regenerative medicine is to ameliorate irreversible destruction of brain tissue by utilizing stem cells to control the process of neurogenesis. The engraftment and survival of AFSCs within the rodent brain was demonstrated in the twitcher mouse model of neurologic disease. ${ }^{1}$ These mice are deficient in the lysosomal enzyme galactocerebrosidase and undergo extensive neurodegeneration and neurologic deterioration, initiating with the dysfunction of oligodendrocytes. AFSCs implanted directly into the lateral ventricles of the developing brain of a newborn mouse survive and integrate into the fetal mouse brain, with $>70 \%$ of administered cells surviving 2 months following implantation. Rehni et al highlighted the potency of AFSCs in a mouse model of ischemic stroke. ${ }^{55}$ In this model, middle cerebral artery occlusion and reperfusion produces ischemia and reperfusion-induced cerebral injury and induces behavioral deficits in mice. Behavioral changes included markedly impaired memory, motor coordination, sensorimotor ability, and somatosensory functions. Intracerebroventricular administration of AFSCs had a significant neuroprotective effect, reversing the focal cerebral ischemia-reperfusion induced behavioral deficits observed in untreated mice. These studies suggest that AFSCs may have important clinical applications for the treatment of degenerative or behavioral brain disorders, paving the way for potential treatments for disease such as stroke, Parkinson disease, Alzheimer disease, and spinal injuries.

\section{Blood and Immune System Regeneration}

Ditadi and coworkers demonstrated that AFSCs display multilineage hematopoietic differentiation potential, generating erythroid, myeloid, and lymphoid cells in vitro, suggesting that AFSCs may be an important source of cells to regenerate the hematopoietic system. ${ }^{56}$ This study demonstrated that 4 months following AFSC injection into immunodeficient $\mathrm{RAG}^{-1-}$ C57BL/6 (Ly5.1) mice, AFSC-derived macrophages, NK, B, and T cells (both $\mathrm{CD} 4+$ and $\mathrm{CD} 8+$ $\mathrm{CD} 3+$ ) were found in transplanted animals. Secondary transplantation was partially successful, suggesting the presence of a small number of hematopoietic progenitor cells within the multipotent AFSC population. These transplantation experiments indicated that AFSCs possess longterm in vivo hematopoietic repopulating capacity and potential therapeutic applications for the treatment of blood and immune disorders.

\section{Conclusion}

Transplantation of stem cells derived from gestational tissue has the potential to treat a range of conditions ranging from diabetes to neural degeneration. Gestational tissue is an abundant source of highly multipotent anti-inflammatory cells, with minimal ethical and legal considerations involved in their collection and use. In clinical and preclinical studies, gestational stem cells have shown efficacy in the treatment of Crohn disease, lung disease, diabetes, repair of bone defects, heart disease, kidney disease, neural degeneration, and blood disorders. Stem cells derived from the placenta, placental membranes, and amniotic fluid remain an abundant and valuable tool for regenerative therapy. 


\section{References}

1 De Coppi P, Bartsch G Jr, Siddiqui MM, et al. Isolation of amniotic stem cell lines with potential for therapy. Nat Biotechnol 2007; 25(1):100-106

2 Murphy S, Rosli S, Acharya R, et al. Amnion epithelial cell isolation and characterization for clinical use. Curr Protoc Stem Cell Biol 2010;Chapter 1:Unit 1E.6

3 Serikov V, Hounshell C, Larkin S, et al. Human term placenta as a source of hematopoietic cells. Exp Biol Med (Maywood) 2009; 234(7):813-823

4 Troyer DL, Weiss ML. Wharton's jelly-derived cells are a primitive stromal cell population. Stem Cells 2008;26(3):591-599

5 Galende E, Karakikes I, Edelmann L, et al. Amniotic fluid cells are more efficiently reprogrammed to pluripotency than adult cells. Cell Reprogram 2010;12(2):117-125

6 Ilancheran S, Michalska A, Peh G, Wallace EM, Pera M, Manuelpillai U. Stem cells derived from human fetal membranes display multilineage differentiation potential. Biol Reprod 2007;77(3): 577-588

7 In 't Anker PS, Scherjon SA, Kleijburg-van der Keur C, et al. Amniotic fluid as a novel source of mesenchymal stem cells for therapeutic transplantation. Blood 2003;102(4):1548-1549

8 Delo DM, De Coppi P, Bartsch G, Jr, Atala A. Amniotic fluid and placental stem cells. Methods in Enzymology 2006;419:426-438

9 Shaw SW, David AL, De Coppi P. Clinical applications of prenatal and postnatal therapy using stem cells retrieved from amniotic fluid. Curr Opin Obstet Gynecol 2011;23(2):109-116

10 Murphy S, Lim R, Dickinson H, et al. Human amnion epithelial cells prevent bleomycin-induced lung injury and preserve lung function. Cell Transplant 2011;20(6):909-923

11 Furth ME, Atala A. Stem cell sources to treat diabetes. J Cell Biochem 2009;106(4):507-511

12 Delo DM, Olson J, Baptista PM, et al. Non-invasive longitudinal tracking of human amniotic fluid stem cells in the mouse heart. Stem Cells Dev 2008;17(6):1185-1194

13 Perin L, Giuliani S, Jin D, et al. Renal differentiation of amniotic fluid stem cells. Cell Prolif 2007;40(6):936-948

14 Snow MH, Bennett D. Gastrulation in the mouse: assessment of cell populations in the epiblast of tw18/tw18 embryos. J Embryol Exp Morphol 1978;47:39-52

15 Downs KM, Harmann C. Developmental potency of the murine allantois. Development 1997;124(14):2769-2780

16 Downs KM, Hellman ER, McHugh J, Barrickman K, Inman KE. Investigation into a role for the primitive streak in development of the murine allantois. Development 2004;131(1):37-55

17 Gardner RL, Beddington RS. Multi-lineage 'stem' cells in the mammalian embryo. J Cell Sci Suppl 1988;10:11-27

18 Loebel DA, Watson CM, De Young RA, Tam PP. Lineage choice and differentiation in mouse embryos and embryonic stem cells. Dev Biol 2003;264(1):1-14

19 Moser M, Li Y, Vaupel K, et al. Placental failure and impaired vasculogenesis result in embryonic lethality for neuropathy target esterase-deficient mice. Mol Cell Biol 2004;24(4): 1667-1679

20 Smith JL, Gesteland KM, Schoenwolf GC. Prospective fate map of the mouse primitive streak at 7.5 days of gestation. Dev Dyn 1994;201(3):279-289

21 Kinder SJ, Tsang TE, Quinlan GA, Hadjantonakis AK, Nagy A, Tam PP. The orderly allocation of mesodermal cells to the extraembryonic structures and the anteroposterior axis during gastrulation of the mouse embryo. Development 1999;126(21):4691-4701

22 Parameswaran M, Tam PP. Regionalisation of cell fate and morphogenetic movement of the mesoderm during mouse gastrulation. Dev Genet 1995;17(1):16-28

23 Rathjen J, Lake JA, Bettess MD, Washington JM, Chapman G, Rathjen PD. Formation of a primitive ectoderm like cell population, EPL cells, from ES cells in response to biologically derived factors. J Cell Sci 1999;112(Pt 5):601-612
24 Dang SM, Kyba M, Perlingeiro R, Daley GQ, Zandstra PW. Efficiency of embryoid body formation and hematopoietic development from embryonic stem cells in different culture systems. Biotechnol Bioeng 2002;78(4):442-453

25 Li L, Arman E, Ekblom P, Edgar D, Murray P, Lonai P. Distinct GATA6and laminin-dependent mechanisms regulate endodermal and ectodermal embryonic stem cell fates. Development 2004;131 (21):5277-5286

26 Kaviani A, Perry TE, Dzakovic A, Jennings RW, Ziegler MM, Fauza DO. The amniotic fluid as a source of cells for fetal tissue engineering. J Pediatr Surg 2001;36(11):1662-1665

27 Robinson WP, McFadden DE, Barrett IJ, et al. Origin of amnion and implications for evaluation of the fetal genotype in cases of mosaicism. Prenat Diagn 2002;22(12):1076-1085

28 Bartha JL, Romero-Carmona R, Comino-Delgado R, Arce F, Arrabal J. Alpha-fetoprotein and hematopoietic growth factors in amniotic fluid. Obstet Gynecol 2000;96(4):588-592

29 Heidari Z, Isobe K, Goto S, Nakashima I, Kiuchi K, Tomoda Y. Characterization of the growth factor activity of amniotic fluid on cells from hematopoietic and lymphoid organs of different life stages. Microbiol Immunol 1996;40(8):583-589

30 Sakuragawa N, Elwan MA, Fujii T, Kawashima K. Possible dynamic neurotransmitter metabolism surrounding the fetus. J Child Neurol 1999;14(4):265-266

31 Srivastava MD, Lippes J, Srivastava BI. Cytokines of the human reproductive tract. Am J Reprod Immunol 1996;36(3):157-166

32 Baschat AA, Hecher K. Fetal growth restriction due to placental disease. Semin Perinatol 2004;28(1):67-80

33 Medina-Gómez P, del Valle M. Cultivo de célas de líquido amniótico. Análisis de colonias, metafases e índice mitótico, con fin de descartar contaminación de células maternas. Ginecol Obstet Mex 1988;56:122-126

34 Pittenger MF, Mackay AM, Beck SC, et al. Multilineage potential of adult human mesenchymal stem cells. Science 1999;284(5411): 143-147

35 Parolini O, Alviano F, Bagnara GP, et al. Concise review: isolation and characterization of cells from human term placenta: outcome of the first international Workshop on Placenta Derived Stem Cells. Stem Cells 2008;26(2):300-311

36 Steigman SA, Fauza DO. Isolation of mesenchymal stem cells from amniotic fluid and placenta. Curr Protoc Stem Cell Biol 2007; Chapter 1:Unit 1E.2

37 Tsai MS, Lee JL, Chang YJ, Hwang SM. Isolation of human multipotent mesenchymal stem cells from second-trimester amniotic fluid using a novel two-stage culture protocol. Hum Reprod 2004;19(6):1450-1456

38 Alviano F, Fossati V, Marchionni C, et al. Term amniotic membrane is a high throughput source for multipotent mesenchymal stem cells with the ability to differentiate into endothelial cells in vitro. BMC Dev Biol 2007;7:11

39 Bailo M, Soncini M, Vertua E, et al. Engraftment potential of human amnion and chorion cells derived from term placenta. Transplantation 2004;78(10):1439-1448

40 Li CD, Zhang WY, Li HL, et al. Mesenchymal stem cells derived from human placenta suppress allogeneic umbilical cord blood lymphocyte proliferation. Cell Res 2005;15(7):539-547

41 Kern S, Eichler H, Stoeve J, Klüter H, Bieback K. Comparative analysis of mesenchymal stem cells from bone marrow, umbilical cord blood, or adipose tissue. Stem Cells 2006;24(5):1294-1301

42 Miki T, Marongiu F, Ellis E, Strom SC. Isolation of amniotic epithelial stem cells. Curr Protoc Stem Cell Biol 2007;Chapter 1: Unit 1E.3

$43 \mathrm{Li} \mathrm{H}$, Niederkorn JY, Neelam S, et al. Immunosuppressive factors secreted by human amniotic epithelial cells. Invest Ophthalmol Vis Sci 2005;46(3):900-907

44 Hori J, Wang M, Kamiya K, Takahashi H, Sakuragawa N. Immunological characteristics of amniotic epithelium. Cornea 2006; 25(10, Suppl 1):S53-S58 
45 Kaviani A, Perry TE, Dzakovic A, Jennings RW, Ziegler MM, Fauza DO. The amniotic fluid as a source of cells for fetal tissue engineering. J Pediatr Surg 2001;36(11):1662-1665

46 Hoffman LM, Carpenter MK. Characterization and culture of human embryonic stem cells. Nat Biotechnol 2005;23(6):699-708

47 Guo CS, Wehrle-Haller B, Rossi J, Ciment G. Autocrine regulation of neural crest cell development by steel factor. Dev Biol 1997; 184(1):61-69

48 National Institutes of Health. A multi-center study to evaluate the safety and efficacy of intravenous infusion of human placentaderived cells (PDA001) for the treatment of adults with moderateto-severe Crohn's disease. Available at: http://clinicaltrials.gov/ ct2/show/NCT01155362?term=PDA001\&rank=4

49 Murphy SV, Shiyun SC, Tan JL, et al. Human amnion epithelial cells do not abrogate pulmonary fibrosis in mice with impaired macrophage function. Cell Transplant 2012; April 10 (Epub ahead of print)

50 Wei JP, Zhang TS, Kawa S, et al. Human amnion-isolated cells normalize blood glucose in streptozotocin-induced diabetic mice. Cell Transplant 2003;12(5):545-552
51 Chang CM, Kao CL, Chang YL, et al. Placenta-derived multipotent stem cells induced to differentiate into insulin-positive cells. Biochem Biophys Res Commun 2007;357(2):414-420

52 Bollini S, Cheung KK, Riegler J, et al. Amniotic fluid stem cells are cardioprotective following acute myocardial infarction. Stem Cells Dev 2011;20(11):1985-1994

53 Lee WY, Wei HJ, Lin WW, et al. Enhancement of cell retention and functional benefits in myocardial infarction using human amniotic-fluid stem-cell bodies enriched with endogenous ECM. Biomaterials 2011;32(24):5558-5567

54 Perin L, Sedrakyan S, Giuliani S, et al. Protective effect of human amniotic fluid stem cells in an immunodeficient mouse model of acute tubular necrosis. PLoS ONE 2010;5(2):e9357

55 Rehni AK, Singh N, Jaggi AS, Singh M. Amniotic fluid derived stem cells ameliorate focal cerebral ischaemia-reperfusion injury induced behavioural deficits in mice. Behav Brain Res 2007; 183(1):95-100

56 Ditadi A, de Coppi P, Picone O, et al. Human and murine amniotic fluid c-Kit + Lin- cells display hematopoietic activity. Blood 2009;113(17):3953-3960 